\title{
D. Suresh Kumar: Herbal Bioactives and Food Fortification: Extraction and Formulation
}

\author{
John Edmondson ${ }^{1}$
}

(C) Springer-Verlag Berlin Heidelberg 2015

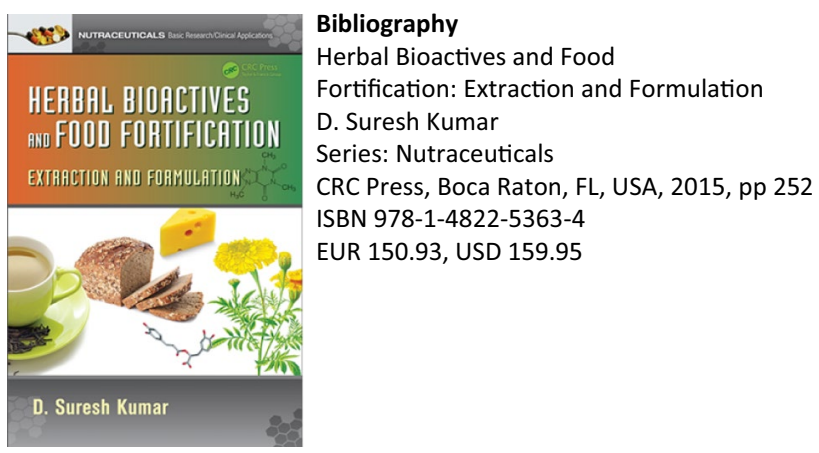

This, the first book in a series on "Neutraceutical: basic research/clinical applications", edited by Kumar takes an unconventional approach to the extraction and formulation of bioactive compounds from plants. It is underpinned by traditional Indian herbal medicine, often referred to as Ayurveda and Siddha Medicine. Writing about a fast-growing market for food supplements, the author assumes little or no prior knowledge of the subject; yet covers extraction techniques in considerable detail. The ultimate purpose seems to be to make food healthier, which given the food and drinks industry's appetite for salt, sugar and ethanol is a commendable objective.

The book is in six chapters. The first is a brief history of medical herbalism, emphasising the proven benefits of neutraceuticals. The second chapter describes the various classes of bioactive substances found in plants, their adaptive significance for the plant and the variability of content due to various environmental factors. The end of the chapter strays into the topic covered by the following one, namely modern methods of extraction and studies of the stability of the resulting compounds. The fourth chapter covers supercritical fluid extraction and highlights some of the difficulties attending this method including its high capital cost. The next and for me the most interesting chapter is titled 'Formulation of food supplements' where rather than incorporating neutraceuticals into foods themselves they are supplied as tablets or capsules. The final chapter deals with food fortification and its impact on such matters as shelf-life and microbiology.

It is difficult to discern an obvious target audience for this book. It undoubtedly summarises the literature in exemplary fashion, and highlights many of the hazards attendant upon the processing and quality control of herbal extracts. At the same time it touches on religious aspects of food certification (Kosher and Halal). I looked in vain for any reference to food allergies in the index, but I may have overlooked some incidental references in the text. As the source of some fascinating anecdotes on modern uses for herbal extracts, it can be recommended: who would guess that immunity-enhancing chewing gum incorporating an extract of Echinacea purpurea would be among the novel products of this industry? The book will be of particular value to people working in the professional field of food ingredient testing and formulation, but for more detailed information on particular products or processes they would need to refer to the wider literature.

John Edmondson

a.books@mac.com

1 Heswall, Cheshire, UK 